\title{
Hippocampal Malrotation is Associated with Chromosome 22q11.2 Microdeletion
}

\author{
Danielle M. Andrade, Timo Krings, Eva W.C. Chow, Tim-Rasmus Kiehl, \\ Anne S. Bassett
}

\begin{abstract}
Background: Patients with chromosome 22q11.2 deletion syndrome (22q11DS) are at a seven fold increased risk of developing seizures. However, only a fraction of these patients exhibit structural abnormalities such as polymicrogyria (PMG) and periventricular nodular heterotopia (PNH) that are known to cause seizures and to be associated with 22q11DS. In this study we used a dedicated seizure imaging protocol to look for additional structural abnormalities in these individuals that may explain the elevated risk of seizure disorder in this patient group. Methods: Nineteen consecutive adult subjects with 22q11DS underwent a 3 Tesla MRI with a dedicated high-resolution seizure protocol. Neurological exam was performed in all patients. Genome-wide analysis excluded the presence of other pathogenic microdeletions or duplications. Results: Structural abnormalities were found in 11 of 14 subjects with sufficient image quality. These included three patients with PNH, one of whom had associated PMG. In addition, there was a surprisingly high prevalence of unilateral hippocampal malrotation (HIMAL), observed in 9 of 14 cases (64\%). EEG findings showed interictal epileptiform discharges with focal distribution in four patients and generalized discharges in one patient. Conclusion: The results suggest that, in addition to other known structural abnormalities, 22q11DS is associated with HIMAL. It has been suggested that this developmental abnormality of the hippocampus may predispose or otherwise contribute to epileptogenesis. However in this study we observed HIMAL in a large proportion of patients, with and without epilepsy. Therefore, other as yet unknown factors may contribute to the high prevalence of epilepsy in this population.
\end{abstract}

RÉSUMÉ: La malrotation de l'hippocampe est associée à une microdélétion 22q11.2. Contexte : Les patients atteints du syndrome associé à la délétion située sur le chromosome 22q11,2 (22q11DS) ont un risque 7 fois supérieur de présenter des crises d'épilepsie. Cependant, seulement un petit nombre de ces patients présentent des anomalies structurales telles la polymicrogyrie (PMG) et l'hétéropie nodulaire périventriculaire (HNP) qui sont des causes connues de crises convulsives et sont associées à 22q11DS. Dans cette étude, nous avons eu recours à un protocole d'imagerie spécifique pour mettre en évidence les crises convulsives afin de tenter de mettre en évidence d'autres anomalies structurales qui pourraient expliquer le risque élevé de désordre convulsif chez ce groupe de patients. Méthode : Dix-neuf sujets adultes consécutifs porteurs de 22q11DS ont subi une IRM 3 Tesla utilisant un protocole spécifique à haute résolution pour les crises convulsives. Un examen neurologique a été effectué chez tous les patients. Une analyse génomique a exclu la présence d'autres microdélétions ou duplications pathogènes. Résultats : Des anomalies structurales ont été détectées chez 11 des 14 sujets dont la qualité de l'imagerie était adéquate. Trois de ces patients étaient porteurs de HNP dont un avait également une PMG. De plus, à notre grande surprise, la prévalence d'une malrotation unilatérale de l'hippocampe (MALHI) était élevée, soit chez 9 des 14 patients (64\%). Les constatations faites à lÉEG incluent des décharges épileptiformes interictales à distribution focale chez 4 patients et des décharges généralisées chez un patient. Conclusion : Selon ces résultats, 22q11DS est associé à une MALHI, en plus des autres anomalies structurales connues. Il a été proposé que cette anomalie du développement de l'hippocampe puisse prédisposer, ou à tout le moins contribuer, à l'épileptogenèse. Cependant, dans cette étude nous avons observé une MALHI chez une grande proportion de patients avec ou sans épilepsie. D'autres facteurs encore inconnus pourraient donc contribuer à la prévalence élevée d'épilepsie dans cette population de patients.

Can J Neurol Sci. 2013; 40: 652-656

Microdeletion at chromosome 22q11.2 is the most common pathogenic copy number variation (CNV) in humans, affecting $1 / 4000$ live births ${ }^{1,2}$. Chromosome 22 has a region particularly susceptible to chromosomal rearrangements due to the presence of several areas of low copy repeats. Hemizygous deletions in this 22q11.2 region, most commonly involving $3 \mathrm{Mb}^{3,4}$ and encompassing more than 45 genes $^{5}$, are responsible for $22 \mathrm{q} 11.2$ deletion syndrome (22q11DS). Patients with 22q11DS are clinically very complex with multisystem abnormalities. There are over 40 common clinical features ${ }^{6}$. Typical features include learning disabilities, subtle dysmorphic facies, velopharyngeal insufficiency, and congenital heart disease. Hypocalcemia due to parathyroid dysfunction are also very common ${ }^{6-8}$. Schizophrenia develops in up to $25 \%$ of patients ${ }^{9,10}$.
Epilepsy is 7 times more common in patients with 22q11DS than the general population ${ }^{11,12}$. Non-hypocalcemic seizures in 22q11DS patients can have generalized or partial (i.e. focal) onset. According to Kao et al, partial onset seizures affect $5.5 \%$

From the Division of Neurology (DMA), Department of Radiology (TK), Krembil Neuroscience Program, Department of Psychiatry (EWCC, ASB), Department of Laboratory Medicine and Pathobiology (TRK), University of Toronto, Toronto Western Hospital; Clinical Genetics Research Program (EWCC, ASB), Centre for Addiction and Mental Health; Department of Pathology (TRK), Division of Cardiology (ASB),

Department of Medicine, University Health Network, Toronto, Ontario, Canada

Received February 13, 2013. Final Revisions Submitted April 29, 2013. Correspondence to: Danielle Andrade at 5W-445, Toronto Western Hospital,

University of Toronto, Toronto, Ontario, M5T 2S8, Canada.

Email: danielle.andrade@uhn.ca 
of all patients with $22 q 11 D S^{11}$. Known potential causes of partial onset seizures in this group of patients include rare case reports of polymicrogyria $(\mathrm{PMG})^{13}$, periventricular nodular heterotopia $(\mathrm{PNH})^{14}$, or hippocampal atrophy $(\mathrm{HA})^{15}$. However, the prevalence of these abnormalities in the 22q11DS population is low, accounting for, at most, $25 \%$ of all cases of partial onset epilepsy ${ }^{11,12}$

Since there is a discrepancy between the prevalence of partial onset epilepsy and the prevalence of known causal structural abnormalities, the aim of this study was to evaluate a series of subjects with 22q11DS, using high resolution magnetic resonance imaging (MRI) and electroencephalography (EEG) to specifically search for additional findings to explain this difference.

\section{Patients And Methods}

Patients: Nineteen adult patients with 22q11DS consecutively referred between 2009-2011 from an adult cohort enriched for neuropsychiatric disorders ${ }^{4}$ comprised the sample for this study. Inclusion criteria: absence of other pathogenic CNVs. Exclusion criteria: seizures associated with stroke or hypocalcemia. Patients without previous history of seizures were included.
Epilepsy history and assessment was conducted by an experienced epileptologist (DA). Diagnosis of epilepsy and seizure types was made according to the classification of the International League Against Epilepsy (www.ilae.org). Electroencephalogram (EEG) studies: Patients were subjected to prolonged digital EEG recordings lasting between one and seven days. Overnight recordings were obtained either through admission to an Epilepsy Monitoring Unit or through ambulatory EEG. Digital EEG recordings were performed using the international 10-20 system in XLTEK recording equipment.

Genetic studies: 22q11.2 microdeletion was confirmed by FISH analysis using standard probes in all patients ${ }^{4,16}$. All participants also had genome-wide microarray data available to exclude the presence of other pathogenic $\mathrm{CNVs}^{4}$.

Imaging: Patients were scanned on a 3 Tesla magnetic resonance imaging (MRI) system using an eight-channel head coil. We used a dedicated seizure scanning protocol including axial and coronal high resolution T1 IR (TE/TR/TI: 42/6500/170ms; 3mm slice thickness, 512x512 Matrix) and T2 FLAIR (TE/TR/IR/: 136/8650/2250ms, 4mm slice thickness, $384 \times 224$ Matrix ) sequences. Coronal sequences were angulated perpendicular to the hippocampi and caution was taken to

Table: General characteristics of 19 adult patients with $22 q 11.2$ deletion syndrome

\begin{tabular}{|c|c|c|c|c|c|c|c|c|c|c|c|c|c|}
\hline \multicolumn{6}{|c|}{ Demographic and neuropsychiatric characteristics } & \multicolumn{8}{|c|}{ Seizure related findings } \\
\hline & & & & & & & \multicolumn{3}{|c|}{ EEG findings } & \multicolumn{4}{|c|}{ MRI findings } \\
\hline Case \# & Sex & Age $(y)$ & disability & SZ & neuropsych & Seizure type & IED & IS & BA & other & PMG & PNH & HIMAL \\
\hline \multicolumn{14}{|c|}{ Patients with epilepsy } \\
\hline 1 & $\mathrm{~F}$ & 23 & Borderline & $\sqrt{ }$ & ADHD & GTCS and MS & 3-4Hz G-PWS & & $\mathrm{N}$ & $\checkmark$ & & & $\sqrt{ }$ \\
\hline 2 & $\bar{M}$ & 58 & Mild & $\mathbf{O}$ & & $2^{\circ} \mathrm{GTCS}$ & & BT & $\mathrm{S}$ & & & & \\
\hline 3 & $\bar{M}$ & 36 & Severe & $\sqrt{ }$ & OCD & CPS and $2^{\circ}$ GTCS & RT, LFC & & $\mathrm{S}$ & & & & \\
\hline 4 & $\bar{F}$ & 19 & Mild & $\sqrt{ }$ & OCD & CPS and $2^{\circ} \mathrm{GTCS}$ & & $\mathrm{G}$ & $\mathrm{S}$ & & & $\sqrt{\mathrm{RFH}}$ & $\checkmark$ \\
\hline 5 & $\bar{M}$ & 34 & Mild & $\sqrt{ }$ & & CPS and $2^{\circ} \mathrm{GTCS}$ & & $\bar{G}$ & $\mathrm{~N}$ & & & $\sqrt{ } \mathrm{LFH}$ & $\sqrt{ }$ \\
\hline 6 & $\mathrm{~F}$ & 43 & Borderline & $\sqrt{ }$ & & CPS & BT & BT & $\mathrm{N}$ & & $\checkmark$ & $\sqrt{ } \mathrm{LFH}$ & \\
\hline 7 & $\mathrm{M}$ & 24 & mild & & & CPS & $\mathrm{BF}$ & & $\mathrm{S}$ & & & & \\
\hline \multicolumn{14}{|c|}{ Patients without epilepsy } \\
\hline 8 & $\mathrm{~F}$ & 22 & Mild & & MD & & LFC & $\mathrm{BF}$ & $\mathrm{N}$ & $\sqrt{ }$ & & & \\
\hline 9 & $\mathrm{~F}$ & 40 & Borderline & $\sqrt{ }$ & & & & $\overline{\mathrm{BPQ}}$ & $\mathrm{N}$ & $* / \mathrm{WM}$ & & & \\
\hline 10 & $\mathrm{M}$ & 20 & Borderline & & & & & $\mathrm{BT}$ & $\mathrm{N}$ & $* *$ & & & \\
\hline 11 & $\mathrm{M}$ & 19 & Borderline & $\checkmark$ & & & & & $\mathrm{N}$ & $\checkmark$ & & & $\sqrt{ }$ \\
\hline 12 & $\bar{M}$ & 30 & Mild & $\sqrt{ }$ & & & & & $\mathrm{N}$ & $\sqrt{ }$ & & & $\sqrt{ }$ \\
\hline 13 & $\bar{M}$ & 34 & Mild & $\sqrt{ }$ & & & & $\mathrm{G}$ & $\mathrm{N}$ & & & & $\sqrt{ }$ \\
\hline 14 & $\mathrm{~F}$ & 20 & Mild & & MD & & & & $\overline{\mathrm{N}}$ & & & & $\checkmark$ \\
\hline 15 & $\mathrm{~F}$ & 37 & Borderline & & MD, GAD & & & & $\mathrm{N}$ & & & & \\
\hline 16 & $\mathrm{~F}$ & 26 & Borderline & & MD & & & & $\mathrm{N}$ & & & & $\checkmark$ \\
\hline 17 & $\mathrm{M}$ & 21 & Normal & & ASD & & & & $\mathrm{N}$ & $\checkmark$ & & & $\sqrt{ }$ \\
\hline 18 & $\mathrm{M}$ & 37 & Borderline & $\sqrt{ }$ & & & & & $\mathrm{S}$ & $\checkmark$ & & & \\
\hline 19 & $\mathrm{M}$ & 37 & Borderline & $\checkmark$ & OCD & & & & $\mathrm{S}$ & & & & \\
\hline
\end{tabular}

All patients with schizophrenia (SZ) or other psychotic illnesses were receiving antipsychotic medications. Patients with major depression and/or anxiety disorders were receiving antidepressant/antianxiety medications. All patients were either treated for documented hypocalcemia or received prophylactic vitamin $\mathrm{D}$ and calcium supplements for the elevated risk of hypocalcemia in 22q11DS.*: hipocampal atrophy; **: focal scarring over right mid-temporal gyrus; $\mathbb{O}$ : psychosis not otherwise specified; $2^{\circ}$ GTCS: secondarily generalized tonic-clonic seizures; ADHD: attention deficit hyperactivity disorder; ASD: autism spectrum disorder; BA: background activity; BF: bi-frontal; BPQ: both posterior quadrants; BT: bi-temporal; CPS: complex partial seizures; EEG: electroencephalogram; G: generalized; GAD: generalized anxiety disorder; G-PSW: generalized polyspike and waves; GTCS: generalized tonic-clonic seizures; HIMAL: hippocampal malrotation; IED: interictal epileptiform discharges; IS: intermittent slowing; LFC: left fronto-central; LFH: left frontal horn MD: major depression; MRI: magnetic resonance imaging; MS: myoclonic seizures; N: Normal; OCD: obsessive compulsive disorder; PMG: polymicrogyira; PNH: periventricular nodular heterotopia; RFH: right frontal horn; RT: right temporal; S: slow; SZ: schizophrenia, WM: white matter. 


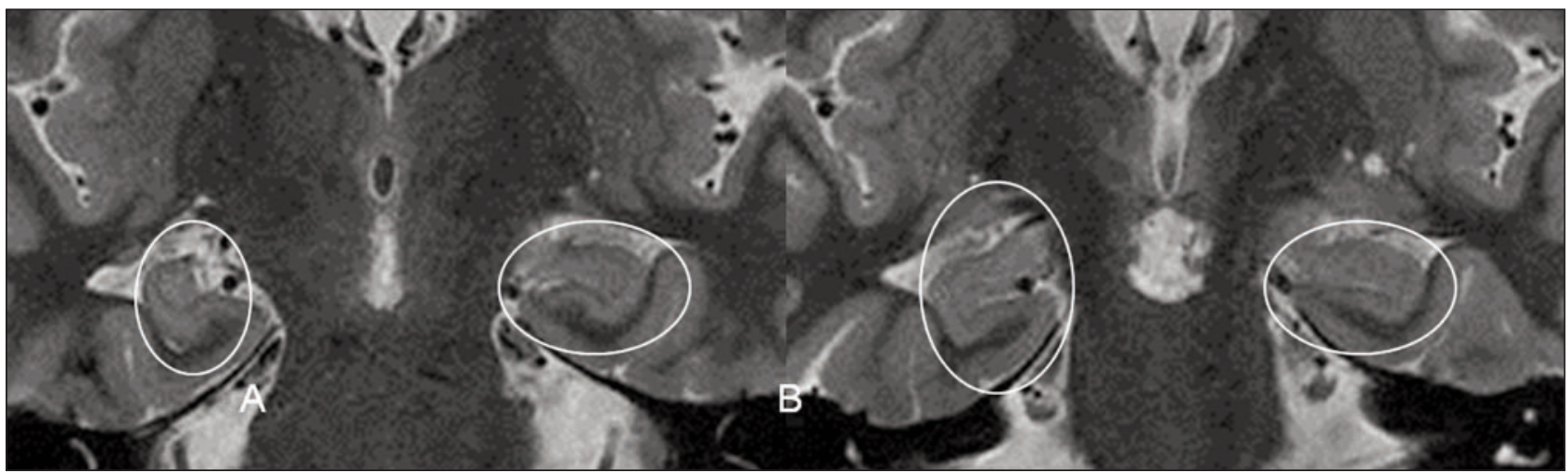

Figure 1: Hippocampal malrotation (HIMAL). $3 T$ MRI of two coronal cuts (A and B) through the anterior third of the hippocampi. Note the right hippocampus (on the left side of the images) has an abnormal vertical orientation. All of the features in the strict characterization of HIMAL proposed by Barsi ${ }^{17}$ are also present: unilateral involvement, incomplete rotation of a hippocampus with abnormal round shape; normal size and signal intensity and blurred inner structure.

include the same AP level of the hippocampus on the same slice. In addition, isotropic sagittal high resolution T1 FSPGR sequences $(1 \mathrm{~mm}$ thickness, isotropic voxels TE/TI/FA: (Min/450/12,1mm slice thickness, 256x256 Matrix)), and standard axial diffusion weighted and $\mathrm{T} 2 *$ weighted gradient echo sequences were obtained.

Image analysis included careful evaluation of the mesial temporal lobe structures including evaluation of the hippocampal rotation. Hippocampal malrotation (HIMAL) was identified based on fulfilling all four Barsi criteria ${ }^{17}$ : 1. unilateral involvement; 2. incomplete rotation of a hippocampus with abnormal round shape; 3 . normal size and signal intensity; 4 . blurred inner structure.

This study was approved by the University Health Network research ethics board.

\section{RESULTS}

Patients' demographics and clinical characteristics are presented in the Table. Nineteen patients between 19-58 (average 30.5) years of age were assessed. Eight were females.

Seizures: Seven patients had a clinical history of recurrent, unprovoked, non-hypocalcemic seizures. EEG did not disclose any interictal epileptiform discharges (IEDs) in two of these patients. One patient had generalized, bisynchronous, 3-4 $\mathrm{Hz}$ frontally predominant polyspike and waves. Four patients had focal IEDs, indicating partial onset epilepsy. Of these four patients with focal IEDs, three had very poor

Figure 2: Prevalence of HIMAL in adult patients with 22 11.2 deletion syndrome from this study compared to the reported prevalence in studies of other conditions. Legend: Prevalence of HIMAL in the following populations: Control: this is represented here (first bar) as the mean of the values from three independent studies: 0/497 controls ${ }^{18}$, 5/50 20 and 19/100 controls $^{19}$. General Epilepsy Population: out of 527 patients with epilepsy (multiple causes) 32 (5\%) had HIMAL ${ }^{17}$. Patients with Prolonged Febrile Seizures: out of 107 children enrolled, $12 \%$ of them had HIMAL ${ }^{21}$. Patients with Other Brain Developmental Abnormalities: The presence of HIMAL in patients with other hemispheric developmental disorders was $22 \% 17$. No genetic tests results were available in the above four categories of patients. Patients with Chromosome 22q11.2 Deletion: without screening for the presence of seizures 9 out of 14 had HIMAL. Patients with Chromosome 22q11.2 Deletion without Epilepsy: 6 out of 10 patients in this group had HIMAL. Patients with Chromosome 22q11.2 Deletion with epilepsy: 3 out of 4 patients in this group had HIMAL. 
quality MRI and no correlation to structural abnormalities could be made. One patient with IEDs over both frontal and temporal regions had PNH over the left frontal horn and widespread PMG over the right hemisphere.

Structural abnormalities: Five patients moved or were claustrophobic during the MRI study. Therefore we had MRI of sufficient quality for 14 patients. Structural abnormalities that were previously described in patients with 22 q11DS were seen in our group at the following rates: mild to moderate generalized atrophy in seven; PMG in one and $\mathrm{PNH}$ in three patients (the patient with PMG also had PNH). Focal scaring over the middle temporal gyrus in one patient. One patient had HA, without abnormal MRI signal, volume loss of the temporal white matter, or atrophy of the fornix or ipsilateral mammillary bodies to support the diagnosis of hippocampal sclerosis.

However, in addition to the abnormalities previously described in the literature, we found HIMAL in nine $(64 \%)$ of the 14 patients with 22q11DS and good quality imaging (Figure $1)$. Of the three patients with malformations of cortical development (PMG and PNH), two also had HIMAL (66\%). Of the 10 patients without epilepsy, six had HIMAL (60\%). Of the four patients with epilepsy and good quality MRI, three had HIMAL (75\%) (Figure 2) $)^{17-21}$.

Although this study was designed to look for causes of epilepsy in the 22q11DS population, we observed HIMAL in $50 \%$ of the patients with schizophrenia and $41 \%$ of those with mild or borderline intellectual disability.

\section{DISCUSSION}

Chromosome 22q11.2 microdeletion leads to a complex phenotype with several neurological problems. Different structural abnormalities have been described in this population: generalized brain atrophy ${ }^{22}$, reduced volumes of parietal and temporal lobes ${ }^{23}$, hippocampus ${ }^{24}$, cerebellum and vermis ${ }^{25}$, and thalami ${ }^{26}$, as well as basal ganglia hypertrophy ${ }^{27,28}$. Known structural changes that can be associated with seizures in this population include: $\mathrm{HA}^{15}, \mathrm{PMG}^{13}$ and $\mathrm{PNH}^{14}$. However the low prevalence of HA, PMG and PNH in 22q11DS is insufficient to explain why up to $7 \%$ of these patients have seizures. Interestingly our results revealed for the first time, that HIMAL, a potentially epileptogenic abnormality $17,20,29,30$ is overrepresented in patients with this 22q11DS.

Hippocampal malrotation is a failure of the hippocampus to properly fold around the hippocampal sulcus into the temporal lobe. This process is completed by the 25 th gestational week ${ }^{31}$. Hippocampal malrotation has been mainly described in patients with epilepsy. HIMAL was observed in $2-54 \%$ of patients with seizures associated with other developmental abnormalities, such as absence of corpus callosum, focal cortical dysplasia, hemimegalencephaly, subcortical laminar heterotopia, single or multiple unilateral or bilateral periventricular nodular heterotopia, schizencephaly, and polymicrogyria ${ }^{32}$. A large survey of 527 adult patients with epilepsy showed that $5 \%$ of them had HIMAL ${ }^{17}$. Subsequent studies observed HIMAL in $12 \%$ of patients with prolonged febrile seizures ${ }^{21}$ (Figure 2). However data on large rare CNVs were not reported for these individuals.

The incidence of HIMAL in control populations (without seizures or malformations of cortical development) has been reported to vary between $5 / 50^{20}$ and $19 / 100^{19}$. However, when the stricter Barsi MRI criteria are applied ${ }^{17}$, Gamss and colleagues found that none of the 497 patients without seizures fulfilled all the criteria for HIMAL ${ }^{18}$. Using the same criteria in our cohort, HIMAL was observed in six of ten patients $(60 \%)$ without seizures or malformations of cortical development and good quality MRI ( 6 out of 10 versus 0 out of 497, Fisher's exact $\mathrm{p}<0.001$ ). In those patients with epilepsy (focal or generalized, but none with "temporal lobe epilepsy") and good quality MRI, HIMAL was observed in $75 \%$ of cases. These numbers suggest that the hemizygous deletion of the $22 \mathrm{q} 11.2$ region predisposes to HIMAL.

We propose that HIMAL results from the haploinsufficiency of one or more genes in the $22 \mathrm{q} 11.2$ microdeletion region. If this is true, why do all patients with 22q11DS not have HIMAL? The phenotypic variability that can result from a shared genetic abnormality may be explained by modulatory effects of other variants located elsewhere in the genome. Alternatively, the hemizygosity may unmask a recessive mutation in the other 22q11.2 allele. Other possibilities have also been proposed for phenotypic variability in 22q11DS ${ }^{33}$.

Despite some statistical evidence of the association between HIMAL and epilepsy, the mechanism by which HIMAL leads to or is associated with epilepsy is not well understood. HIMAL is probably not the single reason leading to the development of seizures. However it can be a "first hit" that, in conjunction with other genetic or non-genetic factors may act in concert for the development or maintenance of seizures ${ }^{29}$. HIMAL has also been associated with complex pre-frontal dysfunction in children with epilepsy ${ }^{34}$ and has been observed in adults with schizophrenia $^{35}$. Whether or not HIMAL is also associated with the other neuropsychiatric conditions seen in 22q11DS, e.g., intellectual disability, remains to be determined.

Temporal lobe and specifically hippocampal abnormalities have been previously described in patients with 22q11DS, however HIMAL has never been reported in this population. We believe the reason for this is that HIMAL is much better appreciated with the imaging protocol used in this study. This is a protocol designed for patients with temporal lobe epilepsy and therefore the coronal images on the 3 Tesla magnet allow better resolution and thus identification of this abnormality.

Limitations: The population studied here were from an adult 22q11DS cohort enriched for neuropsychiatric conditions ${ }^{4}$. All these patients were initially seen in the psychiatry clinic and then referred to the epilepsy clinic. This may explain the higher prevalence of seizure disorders $(26 \%)$ in our study compared to a large group of unselected younger patients with 22q11DS (7\% in Kao and others 2004). Despite this, the prevalence of HIMAL observed was much higher than in patients with other forms of epilepsy or febrile seizures, both of which also represent preselected populations. It will be important to study a larger, randomly selected group of patients with 22q11DS with a similar MRI protocol to determine the overall prevalence of HIMAL. Another limitation is that this study was designed to determine the causes of seizures. Therefore the potential relationship between HIMAL and other neuropsychiatric abnormalities such as learning disabilities or schizophrenia will require further studies. 


\section{Conclusion}

Hippocampal malrotation is overrepresented in patients with 22q11DS. Although HIMAL has been previously associated with seizures, its presence in $60 \%$ of $22 q 11 D S$ patients without seizures (and $75 \%$ of those with seizures) suggest that other factors, as yet unknown, are also contributing to the increased epileptogenesis seen in this population. The role of HIMAL in the other neuropsychiatric abnormalities seen in this population will require further study.

\section{ACKNOWLEDGEMENTS}

DA received research grants from Physicians Services Incorporated and Ontario Brain Institute. Other grant support was received from the Canadian Institutes of Health Research (MOP-79518, MOP-89066, and MOP-97800) and Canada Research Chair in Schizophrenia Genetics and Genomic Disorders (ASB).

\section{REFERENCES}

1. Goodship J, Cross I, LiLing J, Wren C. A population study of chromosome 22q11 deletions in infancy. Arch Dis Child. 1998; 79:348-51.

2. Scambler PJ. The 22q11 deletion syndromes. Hum Mol Genet. 2000;9:2421-6.

3. Driscoll DA. Molecular and genetic aspects of DiGeorge/ velocardiofacial syndrome. Methods Mol Med. 2006;126:43-55.

4. Bassett AS, Marshall CR, Lionel AC, Chow EW, Scherer SW. Copy number variations and risk for schizophrenia in 22q11.2 deletion syndrome. Hum Mol Genet. 2008;17:4045-53.

5. Weksberg R, Stachon AC, Squire JA, et al. Molecular characterization of deletion breakpoints in adults with $22 \mathrm{q} 11$ deletion syndrome. Hum Genet. 2007;120:837-45.

6. Bassett AS, Chow EW, Husted J, et al. Clinical features of 78 adults with 22q11 Deletion Syndrome. Am J Med Genet. 2005;138: 307-13.

7. Shprintzen RJ, Goldberg RB, Lewin ML, et al. A new syndrome involving cleft palate, cardiac anomalies, typical facies, and learning disabilities: velo-cardio-facial syndrome. Cleft Palate J. 1978;15:56-62.

8. Matsuoka R, Kimura M, Scambler PJ, et al. Molecular and clinical study of 183 patients with conotruncal anomaly face syndrome. Hum Genet. 1998;103:70-80.

9. Murphy KC, Jones LA, Owen MJ. High rates of schizophrenia in adults with velo-cardio-facial syndrome. Arch Gen Psychiatry. 1999;56:940-5

10. Cohen E, Chow EW, Weksberg R, Bassett AS. Phenotype of adults with the 22q11 deletion syndrome: a review. Am J Med Genet. 1999;86:359-65.

11. Kao A, Mariani J, McDonald-McGinn DM, et al. Increased prevalence of unprovoked seizures in patients with a $22 \mathrm{q} 11.2$ deletion. Am J Med Genet. 2004;129A:29-34.

12. Ryan AK, Goodship JA, Wilson DI, et al. Spectrum of clinical features associated with interstitial chromosome 22q11 deletions: a European collaborative study. J Med Genet. 1997; 34:798-804.

13. Robin NH, Taylor CJ, McDonald-McGinn DM, et al. Polymicrogyria and deletion 22q11.2 syndrome: window to the etiology of a common cortical malformation. Am J Med Genet. 2006; 140:2416-25

14. Kiehl TR, Chow EW, Mikulis DJ, George SR, Bassett AS. Neuropathologic features in adults with 22q11.2 deletion syndrome. Cereb Cortex. 2009;19:153-64.

15. Debbane M, Schaer M, Farhoumand R, Glaser B, Eliez S. Hippocampal volume reduction in 22q11.2 deletion syndrome. Neuropsychologia. 2006;44:2360-5.
16. Driscoll DA, Salvin J, Sellinger B, et al. Prevalence of $22 \mathrm{q} 11$ microdeletions in DiGeorge and velocardiofacial syndromes: implications for genetic counselling and prenatal diagnosis. J Med Genet. 1993;30:813-17.

17. Barsi P, Kenez J, Solymosi D, et al. Hippocampal malrotation with normal corpus callosum: a new entity? Neuroradiology. 2000;42: 339-45.

18. Gamss RP, Slasky SE, Bello JA, Miller TS, Shinnar S. Prevalence of hippocampal malrotation in a population without seizures. AJNR Am J Neuroradiol. 2009;30:1571-3.

19. Bajic D, Wang C, Kumlien E, et al. Incomplete inversion of the hippocampus--a common developmental anomaly. Eur Radiol. 2008;18:138-42.

20. Bernasconi N, Kinay D, Andermann F, Antel S, Bernasconi A. Analysis of shape and positioning of the hippocampal formation: an MRI study in patients with partial epilepsy and healthy controls. Brain. 2005;128:2442-52.

21. Lewis CS, Bello JA. HIMAL is a malformation that predisposes to prolonged febrile seizures: data from FEBSTAT study. Epilepsia. 2006;47 16

22. Eliez S, Schmitt JE, White CD, Reiss AL. Children and adolescents with velocardiofacial syndrome: a volumetric MRI study. Am J Psychiatry. 2000;157:409-15.

23. Eliez S, Blasey CM, Schmitt EJ, White CD, Hu D, Reiss AL. Velocardiofacial syndrome: are structural changes in the temporal and mesial temporal regions related to schizophrenia? Am J Psychiatry. 2001;158:447-53.

24. Deboer T, Wu Z, Lee A, Simon TJ. Hippocampal volume reduction in children with chromosome 22q11.2 deletion syndrome is associated with cognitive impairment. Behav Brain Funct. 2007; 3:54.

25. Eliez S, Schmitt JE, White CD, Wellis VG, Reiss AL. A quantitative MRI study of posterior fossa development in velocardiofacial syndrome. Biol Psychiatry. 2001;49:540-6.

26. Bish JP, Nguyen V, Ding L, Ferrante S, Simon TJ. Thalamic reductions in children with chromosome 22q11.2 deletion syndrome. Neuroreport. 2004; 15:1413-15.

27. Eliez S, Barnea-Goraly N, Schmitt JE, Liu Y, Reiss AL. Increased basal ganglia volumes in velo-cardio-facial syndrome (deletion 22q11.2). Biol Psychiatry. 2002;52:68-70.

28. Kates WR, Burnette CP, Bessette BA, et al. Frontal and caudate alterations in velocardiofacial syndrome (deletion at chromosome 22q11.2). J Child Neurol. 2004;19:337-42.

29. Baulac M, De Grissac N, Hasboun D, et al. Hippocampal developmental changes in patients with partial epilepsy: magnetic resonance imaging and clinical aspects. Ann Neurol. 1998;44:223-33.

30. Matsufuji M, Utsunomiya H, Inoue T, Yasumoto S, Takashima S, Mitsudome A. Magnetic resonance imaging volumetry and clinical analysis of epilepsy patients with unilateral hippocampal abnormality. Pediatr Int. 2012;54:19-26.

31. Bajic D, Ewald U, Raininko R. Hippocampal development at gestation weeks 23 to 36 . An ultrasound study on preterm neonates. Neuroradiology. 2010;52:489-94.

32. Montenegro MA, Kinay D, Cendes F, et al. Patterns of hippocampal abnormalities in malformations of cortical development. J Neurol Neurosurg Psychiatry. 2006;77:367-71.

33. Maynard TM, Gopalakrishna D, Meechan D, Paronett E, Newbern J, Lamantia AS. 22q11 gene dosage establishes an adaptive range for sonic hedgehog and retinoic acid signaling during early development. Hum Mol Genet. 2013;22:300-12.

34. Stiers P, Fonteyne A, Wouters H, D'Agostino E, Sunaert S, Lagae L. Hippocampal malrotation in pediatric patients with epilepsy associated with complex prefrontal dysfunction. Epilepsia. 2010;51:546-55.

35. Connor SE, Ng V, McDonald C, et al. A study of hippocampal shape anomaly in schizophrenia and in families multiply affected by schizophrenia or bipolar disorder. Neuroradiology. 2004;46:52334. 